\title{
Delayed Recanalization of an Embolized Pulmonary Arteriovenous Malformation Caused Brain Abscess in a Patient with Osler-Weber-Rendu Syndrome
}

\author{
Salman Farooq, ${ }^{1}$ Ashkan Mowla, ${ }^{1,}{ }^{*}$ Mahmoud Abdel Razek, ${ }^{1}$ Azher Iqbal, ${ }^{2}$ and Robert N Sawyer ${ }^{1}$ \\ ${ }^{1}$ Stroke Division, Department of Neurology, State University of New York at Buffalo, New York, US \\ ${ }^{2}$ Vascular Interventional Associate, Buffalo General Medical Center, New York, US \\ "Corresponding author: Ashkan Mowla, Stroke Division, Department of Neurology, State University of New York at Buffalo, New York, US. Tel: +716-8597540, Fax: +716-8592430, \\ E-mail: mowla_a@yahoo.com
}

Received 2015 December 19; Revised 2016 January 14; Accepted 2016 March 05.

Keywords: Recanalization, Pulmonary Arteriovenous Malformation, Brain Abscess, Osler-Weber-Rendu Syndrome

Dear Editor,

Osler-Weber-Rendu Syndrome (OWRS), also known as hereditary hemorrhagic telangiectasia, is a rare genetic disorder characterized by telangiectasias and excessive bleeding. Brain abscess and ischemic stroke are rare but potentially serious complications of OWRS, caused by paradoxical embolism from pulmonary arteriovenous malformations (AVMs) (1). We describe a case of a 56 year old woman with past medical history of OWRS who presented to emergency room with headache and confusion and was found to be febrile. She had a known pulmonary AVM, previously coiled. A head CT scan without contrast showed an area of hypodensity of right temporal lobe. Brain MRI revealed a brain abscess (Figure 1) which was treated with antibiotic therapy. A pulmonary angiogram revealed recanalized AVM in the left lobe of lung which was embolized 15 years ago (Figure 2). She underwent endovascular reembolization to avoid future neurologic complications. Frequent follow-up of treated patients is indicated since pulmonary AVM tend to increase in size over time and might cause neurological complications.

\section{Acknowledgments}

None declared.

\section{Footnotes}

Authors' Contribution: All authors have contributed equally, read and approved the submitted manuscript.

Financial Disclosure: Authors have no relevant financial interest to disclose.

Funding/Support: None declared.

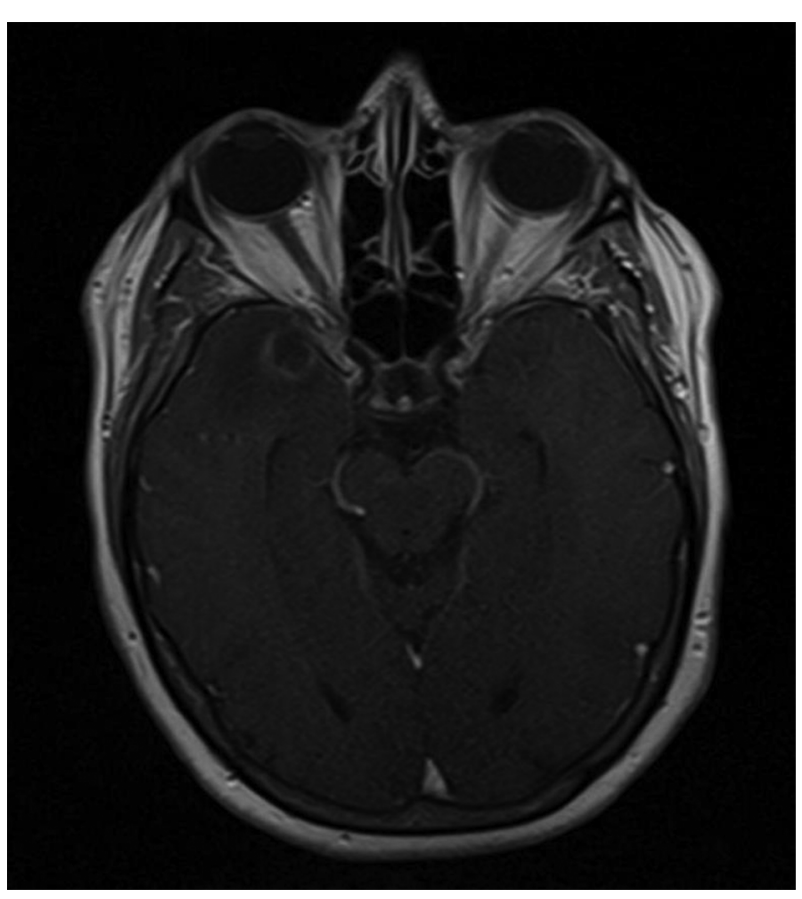

Figure 1. Gadolinium-Contrasted axial T1-Weighted Image of Brain MRI Shows Ring Enhancing Lesion in Right Temporal Lobe With Surrounding Edema 


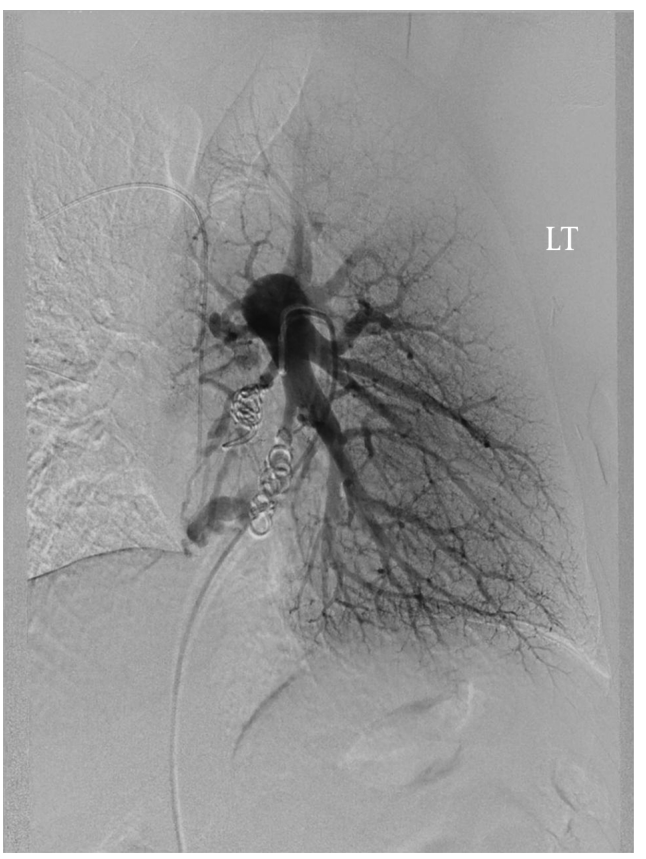

\section{References}

1. Swanson KL, Prakash UB, Stanson AW. Pulmonary arteriovenous fistulas: Mayo Clinic experience. Mayo Clin Proc. 1999:671-80.

Figure 2. Left Lower Lobe Branches of Pulmonary Arteriogram Shows 2 Recanalized AVM Nidi Through the Previously Embolized Feeding Vessel With Microcoils 